\title{
Istilong Voice, Audience, Register at Purpose (VARP) ng mga awitin sa pagsusuring pangnilalaman
}

\author{
Mandado, Juliet O. $\bowtie$ \\ Cebu Technological University-Main Campus, Philippines (ctu2020school@gmail.com)
}

Arnado, Anna Mie M.

Canduman National High School, Philippines (annamie.arnado@deped.gov.ph)

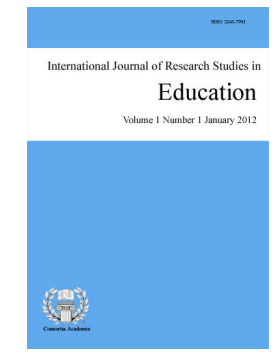

ISSN: 2243-7703 Online ISSN: 2243-7711 DOI: $10.5861 / \mathrm{ijrse} .2021 .5070$

Accepted: 20 January 2021

OPEN ACCESS

\section{Abstract}

Localization in selected Filipino lessons will address one of the missions of basic culture-based education. Just as other countries they promote their language, Filipinos promote their own language in Sugbuanon pinulongan to use as a strategic assembly for an individual ability to use it. The study uses descriptive research and the use of selected popular Visayan songs to achieve the aforementioned using the institutional linguistics of De Matos (1964) which discusses the relationship of language and its user where the style voice, audience, register and Brown's (2016) purpose or VARP that facilitates language teaching and learning from audible and readable information. This study ensures that linguistic content will be answered using 1) Voice- words, phrases, parables: 2) Audience - vocabulary, style of speech; 3) Register- formal and informal: and 4) Purpose - genre and message of songs in society. The study found that the use of local and contextualized lessons is rich in linguistic content in songs translated in the local language and is now trendy music especially in the interest of students because the lyrics of the songs are their vernacular facilitates learning and human relations.

Keywords: voice; purpose; register; audience; content style 


\section{Istilong Voice, Audience, Register at Purpose (VARP) ng mga awitin sa pagsusuring pangnilalaman}

\section{Introduksyon}

Ang lokalisasyon sa mga piling aralin sa Filipino ay tutugon sa isa sa mga misyon ng batayang edukasyon na base sa kultura. Kagaya ng pagtataguyod ng ibang bansa sa kanilang wika ay siya ring pagtataguyod ng mga Pilipino sa sariling wikang Filipino. Ang pinulungan sugbuanon ay isang estratehikong kanya-kanyang kakayahan na ipabatid sa kahit sinoman ang kausap nito.

Pinatotohanan ito ni Pesirla (2012) nang sinabi niyang: "nilangkub ang kahanás istratikahun sa mga istratihiya nga pangpakigpulungay nga gisulti ug wa gisulti”. Nangangahulugan lamang na mayaman ang sa sariling lokal na panitikan ang kultura ng mga Pilipino. Marami ang paraan ng paghahatid ng mensahe sa kapwa, ito ay maaaring sa pamamagitan ng mga berbal at di-berbal na komunikasyon. Isang halimbawa ang musika tungo sa tagapakinig nito. Ang pagkaunawa sa damdaming nais ipabatid mula sa napakinggan ay naaayon sa mga karanasan. Isa sa mga epektibo at makapangyarihang kasangkapang pandamdamin ay ang musika. Ayon kay Cabangon (2016) isang kilalang kompositor sa bansa na lumilikha ng mga awiting nasusulat sa Filipino na sumasalamin ang awiting Filipino sa buhay, gawain, kultura, pakikibaka, inspirasyon, pangyayari sa kapaligiran, at sa nararamdaman ng mga Pilipino. Anumang awitin mapaluma mapabago hangga't naisasapuso ang kahulugan ay malalim ang mapaghuhugutan at ginagamit sa paglalahad ng isang karanasan ukol mga pangyayari sa bawat paligid.

Sa kabilang banda, ang musika ay hindi isang lenggwahe na naglalarawan sa kung ano talaga ang lipunan, subalit ito ay isang metaporang ekspresyon ng mga damdamin na maiuugnay sa takbo ng lipunan Blacking, (1973). Ayon naman kay Ryback (2016) na ang musika ay maaaring maging makapangyarihang instrumentong pang-emosyonal. Habang nabanggit din sa kanyang tesis ni Berdin (2007) sa awit masusukat ang kamalayan sa pulitika, pag-ibig, pakikibaka at pakikipagsapalaran. Samakatuwid, ang instrumentong ito ay maaaring pagyamanin pang lalo para magamit sa epektibong pagtuturo at sa higit na pagkatuto ng mga mag-aaral gaya na lamang ng pag-aangkop ng mga piling awitin sa loob ng klase, na mag-uugnay sa tanikala ng diwa ng awit at sa katotohanan ng araw-araw na pakikipagsapalaran sa buhay.

Ayon kay Cabangon at Santos (2009) na magandang daluyan ng wika ang musika dahil gumagamit ka ng wikang Filipino, naisasaliw ka pa nito, at sa ganitong pamamaraan ay mas mapapalapit ka sa iyong wika, sapagkat tumatagos sa damdamin. Mahalaga ang wika sapagkat ginagamit ito upang malinaw na maipahayag ang damdamin at kaisipan ng tao na sumasalamin sa kultura at panahong kanyang kinabibilangan. Ang wika ay ang masistemang pag-aayaw-ayaw ng lipon ng mga salita na ginagamit sa paghahatid ng mga kaisipan, damdamin at mga hangarin na tangi sa isang pangkat ng tao sa isang pamayanan o bansa wika ni Badayos (2008).

Ang pag-aaral na ito ay suportado din ng Batas Republika Blg. 10533, Seksyon 10, pagbubuo ng kurikulum na nagsasaad na magbubuo ang DepEd ng disenyo at mga detalye ng kurikulum sa pinabuting batayang edukasyon. Makikipagtulungan ito sa Komisyon sa Lalong Mataas na Edukasyon (CHED) upang magbuo ng magkaugnay na mga kurikulum sa batayan at tersiyaryong edukasyon para sa kasanayang pandaigdig ng mga Pilipinong nagsisipagtapos, matiyak ang kahandaan sa kolehiyo, at maiwasan ang pag-uulit sa klase.

Nakitaan ng mananaliksik na isa sa mga palasak ngayon ay ang mga awiting banyaga na nasa wikang Ingles at ang korean wave ay kinahuhumalingan ng mga kabataang nabibilang sa henerasyong ito kahit na hindi naman lubusang maintindihan ang liriko. Ayon sa pag-aaral ni Fuhr (2016), isang assistant professor sa center for world music sa Germany na ang kanilang music videos ay kumakalat sa iba't ibang kontinente at pinapanood ng 
makailang daan at milyon na ulit. Dito nababahala ang mananaliksik sa kahahantungan ng pagkatuto ng mag-aaral kaya bilang guro sa Filipino ay isang hamon ang pagtuturo ng wika at panitikan sa pag-usbong ng makabagong teknolohiya.

Batid ng mananaliksik ang malaking hamon na kailangang mapagtagumpayan ng isang guro. Kaya sa pamamagitan ng malikhaing pagtuturo ay nagkakaroon din ang mga mag-aaral ng malikhaing pagkatuto at isa ang paggamit ng mga awiting visayan popular sa epektibong paraan para matamo ang mga nabanggit gamit ang institutional linguistics ni De Matos (1964) na tumatalakay sa relasyon ng wika at ng gumagamit nito.

Nilalayon ng pag-aaral na ito na masuri ang Voice, Audience, Register at Purpose sa limang piling awiting visapayan popular, batay sa kasanayang pakikinig ng mga mag-aaral. Ginamitan ng leyenda ang mga awitin para mapadali ang pag-uuri ng mga kanta sa loob ng bawat talahanayan nito. Ang tiniyak sa pag-aaral na ito na mabigyang kasagutan ang mga pangnilalamang pangwika na susuriin gamit ang Voice sa; salita, parilala, talinghaga: Audience sa; talasalitaan, istilo ng pannalita; Register sa; pormal at di' pormal: Purpose sa; genre at mensahe ng mga kanta sa lipunan.

\section{Resulta at pagtalakay}

Sa talahanayan 1 matutunghayan ang pangwikang pangnilalaman ayon sa Voice ng mga Awiting VISPop. Sinuri sa bahaging ito ang mga salita, parirala at ang mga talinghagang taglay ng mga awitin sa pamamagitan ng denotasyon at konotasyon ng bawat awitin. Ang istilo ng pagsusulat ay isang karanasang nakaipaabot ang saloobin ng isang manunulat sa paraang salita na nagbibigay ng anyo ng may katuturan ang pahayag na ang taong ito ay nagsasalita ng may malasakit sa mensahe.

\section{Talahanayan 1}

Pangnilalamang istilo ng awitin gamit ang voice ng VARP

\begin{tabular}{|c|c|c|c|c|}
\hline & Denotasyon & Konotasyon & & \\
\hline (PSTK) & $\begin{array}{l}\text {.. kalipay karon } \\
\text { puro na pagmahay.. }\end{array}$ & $\begin{array}{l}\text { walang } \\
\text { naramdamang ligaya }\end{array}$ & $\begin{array}{l}\text { kasakit } \\
\text { Salitang-ugat: } \\
\text { sakit } \\
\text { Panlapi: ka }\end{array}$ & $\begin{array}{l}\text { matinding } \\
\text { damdamin }\end{array}$ \\
\hline (LABU) & $\begin{array}{l}\text { ang tagubtob sa } \\
\text { akong dughan }\end{array}$ & $\begin{array}{l}\text { puno ng sakit, puro } \\
\text { asa na lamang }\end{array}$ & $\begin{array}{l}\text { giduwaan } \\
\text { Salitang-ugat: } \\
\text { duwa } \\
\text { Panlapi: } \\
\text { gi- at -an }\end{array}$ & $\begin{array}{l}\text { akala totohanin na } \\
\text { ang pag-ibig }\end{array}$ \\
\hline$(\mathrm{SAH})$ & $\begin{array}{l}\text { kausa ra tika } \\
\text { higugmaon }\end{array}$ & $\begin{array}{l}\text { Gihatag ang } \\
\text { kasing-kasing }\end{array}$ & $\begin{array}{l}\text { mahigugma } \\
\text { Salitang-ugat: } \\
\text { gugma } \\
\text { Panlapi: } \\
\text { ma- at -hi- }\end{array}$ & $\begin{array}{l}\text { gagawain ang } \\
\text { lahat-lahat }\end{array}$ \\
\hline (PAR) & $\begin{array}{l}\text { basin maapsan } \\
\text { pako }\end{array}$ & Gahi sa paghigugma & $\begin{array}{l}\text { higugmaon } \\
\text { Salitang-ugat: } \\
\text { gugma } \\
\text { Panlapi: } \\
\text { hi- at -on }\end{array}$ & $\begin{array}{l}\text { araw-araw ay isa } \\
\text { lang ang } \\
\text { mamahalin }\end{array}$ \\
\hline (HHS) & $\begin{array}{l}\text { Kay di ta uyab, } \\
\text { wala'y ikaw ug ako }\end{array}$ & Kusog pa sa orasan & $\begin{array}{l}\text { Naglisod } \\
\text { Salitang-ugat: } \\
\text { lisod } \\
\text { Panlapi: } \\
\text { nag- }\end{array}$ & hindi maaangkin \\
\hline
\end{tabular}


Ang konseptong ginamit sa pagsusuri ay ang istilong voice, audience, register at purpose ni Brown (2016) sa pagsusuring saklaw lamang ang mga pangnilalamang wika sa piling awitin. Ang yugtong ito ay naglalahad ng mga pangnilalamang pangwika na nakuha pailalim sa mga awitin. Makikita rin sa bahaging ito ang mga salita, parirala, talinghaga, konteksto, istilo ng pananalita, pangnilalamang pagpapabatid, salitang pormal at di- pormal, genre ng mga awitin. Sa tulong ng mga talahanayan sa bawat bahagi ay isa-isang sinuri ang mga awitin batay sa mga pangnilalamang pangwika nito.

\subsection{Istilo ng awitin gamit ang Voice ng VARP}

Matutuklasan dito sa representasyon ng mga susmusunod na nilalaman ayon sa istilong voice ng awitin at ilang pangnilalamang wika. Sinuri sa bahaging ito ang mga salita, parirala at ang mga talinghagang taglay ng mga awitin sa pamamagitan ng denotasyon at konotasyon ng bawat awitin.

Salita, kahulugan, talinghaga - Inilalahad sa unang awitin na PSTK ang salitang nagpapalutang sa karanasan at naramdaman ng persona ng awitin. Sa pagpapakahulugan ng salitang "giduwaan", ang ibig sabihin nito ay "pinaglaruan". Sa pamamagitan ng mga salita na ginamit sa kabuuan ng kanta ay naipahayag ng persona sa awitin ang kanyang karanasan sa pag-ibig, ang pang-iiwan sa kanya at ang paglalaro sa kanyang damdamin pati na ang naging pagsisisi sa pagmamahal sa maling tao. Mula sa salitang "giduwaan" ay matutukoy din ang mga pangnilalamang pangwika gaya ng salitang-ugat at panlapi. Ang salitang-ugat ay mga salitang buo ang kilos gaya ng salita na "duwa" samantalang ang panlapi ay isang morpema na ikinakabit sa isang salitang-ugat upang makabuo ng iba pang salita gaya ng gi- at -an na isang unlapi at hulapi.

Ang salita ay yunit ng wika na nagdadala ng kahulugan. Ayon pa kay Fortunato at Valdez (1995) na ang mga ito ang bumubuo sa isang wika na nagsisilbing instrumento sa pagbabago ng pananaw ng kapwa sa reyalidad lalo na kung maayos itong magagamit. Mas pinasidhi pang lalo ng persona ang mga ito ng banggitin ang pariralang “...abi nakog tinud-anay" na nagsasaad na "akala ko totoo". Sa pamamagitan ng mga pariralang ginamit sa kabuuan ng kanta ay naipahayag ng persona ang kanyang hinanaing at pagsusumamo. Ang parirala ay mga lipon ng salita na walang simuno at panaguri na ginagamit lamang sa bahagi ng pangungusap.

Sa ikalawang awitin na LABU ang matinding damdamin ng persona ng awitin ay mararamdaman sa salitang "mahigugma" na ang ibig sabihin ay "nagmahal". Sa kabuuan ng mga salitang ginamit sa kanta ay naipahayag ng persona sa awitin ang kanyang wagas na pagmamahal para sa sinisinta na inaalayan niya ng kanyang puso na hanggang kailanman ay dadamayan.

Matutukoy din ang mga pangnilalamang pangwika gaya ng salitang-ugat at panlapi mula sa salitang "mahigugma". Ang salitang-ugat na "gugma" at ang panlaping ma- at -hi- na kapwa mga unlapi na matatagpuan sa unahan ng salitang-ugat. Mas pinalalim pang lalo ng persona ang kanyang ipinahahayag na damdamin sa awitin ng banggitin nito ang isa sa mga pariralang "buhaton kong tanan-tanan" o "gagawin ko ang lahat-lahat".

Ang mga salita at parirala ayon sa boses ng persona sa awitin ay maiuugnay sa teoryang romantisismo, isang teoryang pampanitikan na inspirasyon at imahinasyon ang instrumento sa pagtuklas ng katotohanan, kabutihan at kagandahan. Nabibigyang diin din dito ang iba't ibang damdaming nakapaloob sa akda.

Ang isa sa mga pahayag ng awitin na "Ang tagubtob sa akong dughan..." ay may denotasyon din na pagpapakahulugan na kaagad na mauunawaan ng tagapakinig. "tagubtob sa dughan" na ang ibig sabihin ay kaba ng dibdib. Ginamit din ng persona ang matalinhagang pagpapahayag gaya ng tayutay na onomatopeya o paghihimig. Ang pahayag na ito ay "malakas na tibok ng aking dibdib" ay halimbawa ng tayutay na onomatopeya o paghihimig, naipahihiwatig dito ang kahulugan sa pamamagitan ng tunog o himig ng mga salita na ang kahulugan ay "malakas na tibok" na sa simpleng pagpapakahulugan ay "kaba".

Habang ang pahayag na "Ako nang gihatag nimo akong kasing-kasing..." o "ibinigay ko na sa iyo ang aking puso" ay nagtataglay ng konotatibong kahulugan at ito ay isang pahayag na hayperbole o pagmamalabis. 
Ang pagmamalabis ay lubhang nagpapakita ng kalabisan na imposibleng mangyari sa kalagayan ng tao, bagay, o pangyayari. Iba pang halimbawa ng hayperbole o pagmamalabis ay ang mga sumusunod: namuti ang kanyang buhok sa kahihintay sa sa iyo, abot hanggang langit ang pagmamahal niya sa akin, mga pahayag ng nagbibigay nang malalim na pagpapakahulugan sa bawat bitaw ng salita.

Sa ikatlong kanta na $\mathrm{SAH}$, ang salitang nagpapalutang sa karanasan at damdamin ng persona ng awitin ay ang salitang "higugmaon" na ang ibig sabihin ay "mamahalin". Mahihinuha ang pag-aasam ng persona para sa isang taong ninanais niyang makasama. Matutukoy din ang mga pangnilalamang pangwika gaya ng salitang-ugat at panlapi mula sa salitang "higugmaon". Ang salitang-ugat na "gugma" at ang mga panlaping hi- na isang unlapi na matatagpuan sa unahan ng salitang-ugat at -on na isang hulapi na matatagpuan sa hulihan. Mas pinagtibay pang lalo ng persona ang kanyang pagpapahayag ng damdamin sa pamamagitan ng mga pariralang "adlaw-adlaw ikaw ra gyud" o "araw-araw ikaw lamang". Ito ay sa teoryang romantisismo pa rin maiuugnay na naglalayong ipamalas ang iba't ibang paraan ng tao sa pag-aalay ng kanyang pag-ibig sa kapwa, bansa at mundong kinalakihan. Ipinapakita rin sa akda na gagawin ng isang nilalang ang lahat upang maipaalam lamang ang kanyang pag-ibig sa taong napupusuan. Ang pahayag na "kausa ra tika higugmaon, kausa ra tika pagahagkon" ay nagtataglay ng denotasyon na pagpapakahulugan. "kausa" na ang ibig sabihin ay "isang beses", "higugmaon" o "mamahalin", at "pagahagkon" o "hahalikan".

Sa kabuuan, batay sa salita, parirala at talinghaga na nakapaloob sa awitin ay maaaninag sa boses ng persona ang wagas na pag-ibig na handang ialay sa sinisinta at ang pagmamahal na walang hangganang ipadarama. Ang isang awitin ay nagmula sa isang tula na nilapatan ng tunog. Ang mga ito ay uri ng tula ayon sa anyong tulang liriko o tula ng damdamin. Nagtataglay ito ng mga karanasan, guniguni, kaisipan at mga pangarap na maaaring nadama ng may-akda o ng ibang tao Rebamonte at Montera (2007).

Ang talinghaga ay mga pahayag o ekspresyon na may malalalalim na salita o may hindi tiyak na kahulugan. Sinasalamin nito ang kagandahan at pagkamalikhain ng sariling wika na siyang nagpapatotoo sa bawat lipunan. Ang bawat salita o pahayag may talinghaga ay nagtataglay ng pagpapakahulugang may denotasyon o konotasyon. Ang kabuuan ng awitin gaya ng pahayag na "Ikaw ang akong kalipay, karon puro na pagmahay..." ay may denotasyon na pagpapakahulugan sapagkat ang mga ginamit na salita ng kompositor ay literal at diretsahan na kung saan ay kaagad na nahahanap sa mga diksyunaryo ang kahulugan ng mga salita gaya ng "ikaw" na ang ibig sabihin ay "ako", "kalipay" na ang ibig sabihin ay "kaligayahan", at "pagmahay" na ang ibig sabihin ay "pagsisisi". Makikita ang pahayag sa bawat awitin tungkol sa pagmamahal na ipinaubaya sa kanyang kapwa.

Ginamit din ng persona ang matalinhagang pagpapahayag gaya ng tayutay na pag-uulit na anapora na dagliang nakikita saanmang bahagi ng mga taludtod o pangungusap sapagkat ang unang titik o unang pantig ng bawat salita, o ang isang salita ay makailang beses na ginamit. Sa pahayag na "kausa ra tika higugmaon, kausa ra tika pagahagkon" o "isang beses lang kita mamahalin, isang beses lang kita hahalikan", ito ay pag-uulit na anapora sapagkat ang pag-uulit ng isang salitang "kausa" ay nasa unahan ng isang sugnay.

Sa pagpapakahulugan naman, may mga nabanggit sa kanta na nasa kaisipang sebwano. Isa sa mga ito ay "kung Mahogany ka kay Narra ko" na naghahambing sa tao sa mahogany at narra na mga punong-kahoy. Sa totoong kahulugan ng pahayag, ito ay nagsasaad na "kung sakaling mahulog ka ay nandito lang ako na sasalo sa iyo". Isang paraan ng pagbubukas ng pakikipag-usap sa isang kilala o hindi gaanong kilalang tao upang ipahayag ang saloobin sa pamamagitan ng pagbigkas ng mga pahayag na sadyang nakatatawag pansin.

Sa kabuuan, batay sa salita, kahulugan at talinghaga na nakapaloob sa awitin ay sa boses ng persona at madadama ang pait na nararamdaman ng mga taong may damdaming pinaglaruan at biglang iniwanan na patuloy pa rin sa paghahanap ng kasagutan sa sarili buhat ng naranasang kabiguan. Sa pahayag nina Abangan, V.C. et al. (2009) ang mga tayutay ang mga "garces of language", mga "deressing of thought" dahil ito ay nagbibigay ng palamuti sa mga pahayag na prosa at nagbibigay ng istilo. 
Mandado, J. O., \& Arnado, A. M. M.

Ipinapahiwatig na ang mga ginamit na mga salita, pahayag at talinghaga sa awitin ay nagtataglay ng sapat na kasanayan at kalamang makapag-integreyt nang angkop at wastong pagpapasya na pahalagahan ang sariling likha at pambansang panitikan batay sa umiiral na kasanayang pampagkatuto sa wika na magamit ito sa pagkatuto at pagtuturo sa loob at labas ng paaralan.

\subsection{Istilo ng awitin gamit ang audience ng VARP}

Inilalarawan sa Talahanayan 2 ang istilo ng audience sa pagpapabatid nito sa mga kahulugan gamit ang awitin sa nilalaman ng awitin.

\section{Talhanayan 2}

Pangnilalamang istilo ng awitin gamit ang audience ng VARP

\begin{tabular}{|c|c|c|c|}
\hline Awitin & Istilo ng Pananalita & Konteksto & Pagpapabatid \\
\hline (PSTK) & Pagtatanong & $\begin{array}{l}\text { pero ngano mang kalit kang nibiya } \\
\text { unsa man diay imong gipangita? }\end{array}$ & $\begin{array}{l}\text { nadaramang sakit dahil } \\
\text { iniiwanan at pinaglaruan }\end{array}$ \\
\hline (LABU) & Pagpapahiwatig & $\begin{array}{l}\text { sa kasadya'g kabalaka } \\
\text { kanunay kang may kasangga } \\
\text { I love you langga }\end{array}$ & $\begin{array}{l}\text { nagmamahal at hindi kailanman } \\
\text { mang-iiwan. }\end{array}$ \\
\hline$(\mathrm{SAH})$ & Pang-aaliw & $\begin{array}{l}\text { sa imong pagkalaagan } \\
\text { nasaag ka sa akong dughan } \\
\text { kay nara ka sa akong heart }\end{array}$ & $\begin{array}{l}\text { hindi masabi-sabi ang } \\
\text { damdamin sa sinisinta na } \\
\text { handang mag-alay ng wagas na } \\
\text { pag-ibig. }\end{array}$ \\
\hline (PAR) & Paglalahad & $\begin{array}{l}\text { dili na ko mag-assume } \\
\text { dili na ko magpada } \\
\text { dili na ko magpatunto nimo nga } \\
\text { paasa } \\
\text { pero atik ra }\end{array}$ & $\begin{array}{l}\text { nagpatuloy na umaasa sa } \\
\text { relasyong simula't sapul ay } \\
\text { wala ng katiyakan. }\end{array}$ \\
\hline (HHS) & Paglalahad & $\begin{array}{l}\text { kay di ta uyab, walay ikaw ug ako } \\
\text { Ha Ha Ha katawa } \\
\text { Ha На Нa Hasula }\end{array}$ & $\begin{array}{l}\text { positibo sa lahat at idaan na } \\
\text { lang sa tawa ang kasawian. }\end{array}$ \\
\hline
\end{tabular}

Istilo ng pananalita, konteksto at pagpapabatid - Dito sa mga nilalaman sa konteksto ay isinasaalang-alang ang ugnayan ng mga elementong linggwistikal at kakayahang pangkomunikatibo ng pagpapahayag para epektibong mahagilap ng tagapakinig ang emosyon, karanasan, at mensaheng hatid ng awitin sa pangkabuuan. Gumagamit ito ng panandang konteksto na maaring mga inuulit na salita o parirala na nagpapalalim lalo sa mensahe tungo sa malinaw na kahulugan ng liriko nito na maaring magsilbing kabuuan ng diskurso ng isang awitin.

Sa awiting PSTK, ito ang panandang konteksto na nagbuod sa kahulugang nakabatay sa pahiwatig ng mga pahayag na ginamit ng persona ng awitin, ang "Pero ngano mang kalit kang nibiya, unsa man diay imong gipangita? Palihog pasabta ko..." na inilahad sa istilong pagtatanong kung bakit nang-iwan na lang bigla na may nilalamang nagpapabatid sa mga tagapakinig tungkol sa sakit na nadarama ng mga taong iniwanan at pinaglalaruan.

Ang kabuuang kontekstong napapaloob sa awiting labyu langga ay mahihinuha sa linya ng awiting "kasadya'g kabalaka, kanunay kang may kasangga, I love you langga..." na inilahad sa istilong pagpapahiwatig na may nilalamang ipinararating sa mga tagapakinig na may mga tao pa ring tunay kung magmahal at hindi kailanman mang-iiwan.

Masisilayan sa kontekstong napapaloob sa awiting SAH ang kabuuan mahihinuha sa linya ng awitin "Sa imong pagkalaagan, nasaag ka sa akong dughan.." inilahad sa istilong pang-aaliw na mahihinuha sa mga liriko ng awitin na may pagpapabatid na ipinararating sa mga tagapakinig na kahit hindi man masabi-sabi ang damdamin sa sinisinta ay naririyan pa rin at handang mag-alay ng wagas na pag-ibig. 
Sa awiting pero atik ra, ipinahayag sa linya n "dili na ko mag-assume, dili na ko magpada, dili na ko magpatunto nimo nga paasa. Pero atik ra..." sa istilong paglalahad ng naging karanasan sa pag-ibig at kabiguan na may nilalamang pagpapabatid na ipinararating sa mga tagapakinig na hindi madali ang nararanasan ng taong patuloy na umaasa sa relasyong simula't sapul ay walang katiyakan.

At sa huling awiting HHS naman, ang kabuuang kontekstong napapaloob ay mahihinuha sa linya na "Kay di ta uyab, walay ikaw ug ako apan kon magselos ko ayaw pagbuot. Sige na lang ta ani'g Ha Ha Ha katawa, Ha Ha Ha Hasula..." sa istilong paglalahad ng naging karanasan sa pag-ibig at kabiguan sa sinisintang may iba ng minamahal na may nilalamang pagpapabatid na ipinararating sa mga tagapakinig na maging positibo at idaan na lang sa tawa anumang sasapiting kasawian.

Sa pangkabuuan, mahalaga ang gampanin ng mga awdyens sa kahit anong akdang pangwika at pampanitikan sapagkat sila ang pinagkukunan ng inspirasyon ng bawat manlilikha ng sining. Nagtataglay ito ng mga karanasan, guniguni, kaisipan at mga pangarap na maaaring nadama ng may-akda o ng ibang tao Rebamonte at Montera (2007).

\subsection{Istilo ng awitin gamit ang register ng VARP}

Ang antas ng wika ay nauuri sa dalawa, ang wikang pormal na mga salitang pamantayan dahil ito ay kinikilala, tinatanggap at ginagamit ng karamihang nakapag-aaral sa wika at ang wikang di' pormal na mga salitang karaniwan at palasak sa mga pang-araw-araw na pakikipag-usap at pakikipagsulatan sa mga kakilala o kaibigan. Batid ni Macnamara (1967) na tumutukoy sa mga pangyayaring nakapagpabugso ng damdamin sa mga pahayag na nakasulat sa paraan ng pagkagamit ng wika.

Matutunghayan sa kasunod na Talahanayan na sa istilong gamit sa awitin ayon sa register ay ang mga wikang narehistro ayon sa pagsulat tungo sa pagkagamit ng may pormalidad at di' pormal ang paghahatid nito sa mga mambabasa at makikinig.

\section{Talhanayan 3}

Pangnilalamang istilo ng awitin gamit ang register ng VARP

\begin{tabular}{|c|c|c|}
\hline Awitin & Pormal na wika & di' Pormal na wika \\
\hline (PSTK) & $\begin{array}{l}\text { magpaabot, maghuwat, } \\
\text { makadungog, pagmahay, } \\
\text { giduwaan, pasabta ko }\end{array}$ & tawa, gugma, forever \\
\hline \multirow[t]{2}{*}{ (LABU) } & mahigugma, kasingkasing & langga, okay, happy-happy, ing-ini \\
\hline & kabalaka, I love you & ka'baw, ni'ng \\
\hline \multirow[t]{2}{*}{$(\mathrm{SAH})$} & nawong, adlaw-adlaw, dughan & ugma, dagway \\
\hline & higugmaon, nalipong, nasaag & $\begin{array}{l}\text { cheat day, diet, excuse me, future wife, baby, heart } \\
\text { si'g, o', g'yud, ko'g, tika'g, maho'g, 'nya }\end{array}$ \\
\hline \multirow[t]{2}{*}{ (PAR) } & maghunahuna, modagan, & move on, ballpen, feelings \\
\hline & $\begin{array}{l}\text { mogukod } \\
\text { gugmang hatag bawi }\end{array}$ & ay'na, taw'ng, g'yud, ko'ng \\
\hline \multirow[t]{2}{*}{ (HHS) } & magselos, pagbuot, mahimo & damgo, katawa, mautro \\
\hline & paghisgot, magkadayun & $\begin{array}{l}\text { promise, FLAMES, pa as if, na-fall, } \\
\text { pa'v may'pa ani'g cheat }\end{array}$ \\
\hline
\end{tabular}

May dalawang uri rin ng wikang pormal, ang pampanitikan na mga salitang matatayog, malalalim, makukulay, at sadyang matataas ang uri. Ikalawang uri naman ay ang pambansa na mga salitang ginagamit sa mga aklat at babasasahing ipinalalabas sa buong kapuluan. Magkaiba man ang paraan ng pagkakabigkas sa ilan sa mga salita ngunit masasabing nabibilang pa rin ito sa wikang pormal sapagkat may magkatulad na kahulugan lamang ang mga ito na nauunawaan ng buong kapuluan na makikinita sa pinagkomparang sinugbuanong taludturan ng awiting pasabta ko inilahad ang mga pormal na ang "magpaabot, maghuwat, makadungog, 
pagmahay, giduwaan, pasabta ko" na ibig sabihin ay ang matinding pag-aantay at pagtatanong na kailngan ng kasagutan. Sa taludtod ng awiting Labyu Langga naman ay ang mga sumusunod: "mahigugma, kasingkasing kabalaka" na may kahulugan na puno ito ng pagmamahal sa kanyang kapwa. Sa SAH naman na "di ko ganahan ug sama nimo kay ikaw ra gyud mismo akong gusto" tungo sa salin na "ayoko ng katulad mo dahil ikaw lang mismo ang aking gusto", "kung adlaw ta, ako ang sabado ug ikaw ang dominggo kay ikaw ang ugma ko" tungo sa salin na "kung araw tayo, ako ang sabado at ikaw ang linggo dahil ikaw ang kinabukasan ko" mga pahayag na may kinalaman sa pag-iibigan na may katotohanan.

Sa taludtod ng awiting pero atik ra naman na "unta karon ako makat-on na nga wa gyud ko'y maani ning gugmang hatag-bawi" tungo sa salin na "Sana ngayon ako ay matuto na wala akong maaani sa pag-ibig na bigay-bawi". Sa panghuling taludtod ng awiting HHS naman na "saypa sa akong mga damgo aduna pay ikaw ug ako" tungo sa salin na "buti pa sa aking panaginip ay mayroong ikaw at ako" inilahad na walang pag-asa sa pag-iibig kundi puno ito ng paasa na lamang sa napupusuan.

Ang ikalawang uri ng antas ng wikang di' pormal naman na mga salitang karaniwan at palasak sa mga pang-araw-araw na pakikipag-usap at pakikipagsulatan sa mga kakilala o kaibigan ay nahahati sa tatlong uri. Una, ang wikang lalawiganin gaya ng mga iilan sa mga salita na hinango mula sa kabuuan ng mga awiting ginamit sa pag-aaral na "kalipay (kaligayahan), pagmahay (pagsisisi), katawa (halakhak), gugma (pag-ibig), giduwaan (pinaglaruan), dughan (dibdib), mahalad (maiaalay), kasing-kasing (puso), langga (minamahal), adlaw-adlaw (araw-araw), ugma (bukas), dagway (mukha), maghuna-huna (mag-isip-isip), modagan (tumakbo), mogukod (maghabol), damgo (panaginip), mahadlok (matakot) at mautro (maulit)". Ang mga ito ay nabibilang sa wikang lalawiganin sapagkat ang mga salita ay hindi pamilyar sa buong kapuluan at saklaw lamang ng tiyak na pook na pinaggagamitan nito. Ang sinugbuanong binisaya ay ang lingua franca ng mga nakatira sa gitnang Visayas, ilang bahagi ng Silangang Visayas at ng ibang parte ng Mindanao.

Isang uri ng wikang di' pormal naman ay ang wikang kolokyal na mga salitang ginagamit sa pang-araw-araw na pakikipagtalastasan ngunit may kagaspangan at pagkabulgar, at kadalasan ay ang pagpapaikli ng mga salita gaya ng mga iilan sa mga salita na hinango mula sa kabuuan ng mga awiting ginamit sa pag-aaral na "ing-ini/ingon ani" na ang ibig sabihin ay ganito; "ka'baw/kahibawo" na ang ibig sabihin ay alam ; "si'g/sige ug" na ang ibig sabihin ay palagi ; "g'yud/gayud" na ang ibig sabihin ay talaga ; " nya/unya" na ang ibig sabihin ay "mamaya" ; "ay'na/ayaw na" na ang ibig sabihin ay huwag na ; "taw'ng/tawo nga" na ang ibig sabihin ay "tao na.." ; "maypa/maayo pa" na ang ibig sabihin ay "buti pa"; at "ani'g/ani ug" na ang ibig sabihin ayon sa pagkakagamit sa kanta ay "ganito".

Ang iba pang di' Pormal naman ay ang wikang balbal na kilala rin bilang salitang kanto o salitang kalye. Narito ang iilan sa mga salita na hinango mula sa kabuuan ng mga awiting nasa Sinugbuanong binisaya na ginamit sa pag-aaral gaya ng "forever, okay, happy-happy, I love you, cheat day, diet, excuse me, future wife, baby, heart, move on, ballpen, feelings, promise, at FLAMES na nabibilang sa isa sa palabuuan ng mga salitang balbal na Englisized Category o iningles pati na ang "pa as if at na-fall" na nabibilang sa palabuuan ng salitang balbal na kung tawagin naman ay Mixed Category o pinaghalu-halo.

Sa pangkabuuan, ang rehistro ng bawat awitin ay nauuri sa kung ano tono at antas ng pormalidad ng isang lenggwahe na iniaayon sa awdyens o tagapakinig na madla alinsabay ng layunin at mensaheng nais na ipaaabot. Sa pag-aaral ni Sandoval (2012) mula sa aklat ng Salindaw ni Pregrino et al. (2012) Varayti at baryasyon ng Filipino, na wika sa komnet, isang bagong rehistrong wikang Filipino ang teorya ng rehistro ay tumitingin sa relasyon ng gamit ng wika sa iba't ibang sitwasyon at anyo nito. Matatagpuan ang komunikasyon sa pamamagitan ng paggamit nito ng Malaya ang wika sa pagpapaabot nito sa pasalita o pasulat na paraang naglalayong matuklasan ang maunawan ang mga ito sa mga sitwasyon, lugar, grupo at anyo. Ayon pa kay Thomason (2001) wala pang makapagpapatunay na ang isang wika ay nadebelop nang mag-isa at hindi naimpluwensyahan ng anumang wika.

Ang genre ay ang paraan ng paghahatid ng mensahe sa tagapakinig para magkaroon ng koneksyon at 
pagkakaunawaan. Sa pamamagitan nito ay maiuugnay ang tanikala ng bawat puso at isipan ng persona ng awitin at ng awdyens na siyang tagapakinig. Makatutulong ito sa pagkatuto at nagpapaganyak ng mag-aaral na hindi madali malimutan ang sariling bernakular, nagpapakilala ng sariling kultura, at identidad ang paggamit ng sariling likha tulad ng mga awiting nabanggit sa panunuri na nagtagtaglay ng kakayahan sa paggamit ng wika.

Masisilayan sa kasunod na talahanyan ang paggamit ng istilong purpose sa pagsusuri ng pangnilalamang wika sa mga genre at ang mensahe sa bawat awiting naranasan.

\section{Talhanayan 4}

Pangnilalamang istilo ng awitin gamit ang purpose ng VARP

\begin{tabular}{|c|c|c|}
\hline Awitin & Genre & Mensahe at kahulugan \\
\hline (PSTK) & Nagsasalaysay & $\begin{array}{l}\text { Sa awitin ang naging karanasan sa pag-ibig, ang kabiguang } \\
\text { naranasan. May layong ipaunawa na hindi madali ang masaktan at } \\
\text { iwananan ng biglaan. Ang makasakit ng damdamin ng iba ay hindi } \\
\text { madali ang pagdaraanan ng taong iyong masasaktan. } \\
\text { Inilahad din sa liriko ng awitin na kahit anong ang damdamin }\end{array}$ \\
\hline (LABU) & $\begin{array}{l}\text { Naglalarawan } \\
\text { Naglalahad }\end{array}$ & $\begin{array}{l}\text { kapag nagmamahal ay kayang gawin para sa taong mahal basta at } \\
\text { magkasama lagi sa lungkot man o ligaya. Ipadama ang presensya } \\
\text { na magiging kasangga sa hirap man o ginhawa. }\end{array}$ \\
\hline$(\mathrm{SAH})$ & $\begin{array}{l}\text { Nagsasalaysay } \\
\text { Nang-aaliw }\end{array}$ & $\begin{array}{l}\text { Isinasalaysay sa awitin ang damdaming di masabi-sabi na walang } \\
\text { patutunguhan. Kaya, matapang sa paglalahad ng saloobin dahil } \\
\text { kailanman ay walang patutunguhan ang damdaming itinatago. }\end{array}$ \\
\hline (PAR) & Nagsasalaysay & $\begin{array}{l}\text { Inilahad ang karanasan sa pag-ibig tungkol sa naging damdamin } \\
\text { niya sa isang tao. Naglalahad ng aral na huwag ipilit ang ayaw at } \\
\text { huwag na lang umasa para hindi na masaktan sa huli. }\end{array}$ \\
\hline (HHS) & Nagsasalaysay & $\begin{array}{l}\text { Inihayag ang kanyang karanasan sa pag-ibig sa isang tao na } \\
\text { mayroong gustong iba. Ang sakit na nararamdaman sa tuwing may } \\
\text { iba pero patuloy pa rin ang pag-antay. Maging positibo at idaan na } \\
\text { lang sa tawa ang lahat. }\end{array}$ \\
\hline
\end{tabular}

Note. *1(PSTK-pasabta ko), 2(LABU-Labyu Langga), 3(SAH-Sa akong heart), 4(PAR-Pero atik ra) at 5(HHS-Hahahasula) mga piling awiting ginamit sa pag-aaral.

Ito ay may layunin na ipaunawa din sa mga tao na hindi madali ang masaktan at iwananan ng biglaan na pati ang pangangapa kung maghihintay pa ba at patuloy na umasa o hindi na ay hindi ganoon kadali. Ang mensahe ay para sa lahat na huwag hayaang makasakit ng damdamin ng iba dahil hindi madali ang pagdaraanan ng taong iyong masasaktan.

Samantala, sa mga awiting PSTK, LABU, SAH, PAR at HHS naman, ang genre ng paghahatid sa tagapakinig ay naglalarawan at naglalahad. Inilahad din sa liriko ng awitin na kayang gawin para sa taong mahal basta at magkasama sa hirap at ginhawa. Ang kahulugan para sa lahat ay nagsasaad na ipadama ang totong pagmamahal sa kapwa tao kahit masakit na nararamdaman pero patuloy parin ang paghangad at umasa. Isang patunay ang pag-aaral ni Maceda (2015) sa pagdinig ng kanta, maaring magunita ng tagapakinig ang katulad na danas ng sarili o kamag-anak o kaibigan o kakilala na maari niyang idagdag sa mga linya ng kanta. Sa paraang ito, lumalago ang kanta at nagiging bahagi ng tradisyon ng taong bayan.

Sa pangkabuuan, nararapat na malinaw kung ano ang ang layunin at ang mensahe na nais iparating nang sa gayon ay magkaroon ng matibay na koneksyon ang awitin at ang tagapakinig. Ang mga awitin na sinuri ay lubhang nakatutulong sa pagkatuto ng mga mag-aaral na isang kagamitan o gamit upang lalong mapanatili ang kahandaan at interes ng mga mag-aaral sa pag-aaral at pagkatuto. Isa ito sa hangaring ng mananaliksik na mapalago at malinang pa ang mga kagamitan sa pagtuturo at pagkatuto gamit ang mga lokal na teksto dahil ito ay isang salalayan na may sapat na kaalaman ang mag-aaral sa tekstong interdisiplinari at nagagamit ang Filipino sa interaksyunal na pakikipagtalastasan. 


\section{Natuklasan}

Batay sa ginagawang pagsusuri natuklasan sa pag-aaral na ito ang istilong varp na ginamit sa pagsusuri ng pangnilalamang wika sa piling awitin ay makahulugan at mayaman sa wika. Pagkatapos ng masusing pag-aanalisa ng mga datos, natuklasan ng mananaliksik sa pag-aaral na ito ang mga sumusunod:

1. Ang pagsusuring istilo sa voice sa salita, parirala, talinghaga ay inaangkop sa bawat kahulugan na nais ipabatid ng awitin ay may kasiningan kawili-kawili at naging marubdob ang awitin sa pangkat ng taong nakikinig.

2. Sa pangwikang pangnilalaman sa konteksto, istilo ng pananalita at pagpapabatid ay epektibong naiparating sa awdyens ang emosyon, karanasan, at mensaheng hatid ng awitin sa pangkabuuan dahil sa mga panandang konteksto na nagsaalang-alang sa ugnayan ng mga elementong linggwistikal at kakayahang pangkomunikatibo ng pagpapahayag.

3. Lumabas ang mga antas ng wika ayon sa ilang pagkaiba sa paraan ng pagkakabigkas ay mapapansin ang pagkakatulad sa baybay at kahulugan nito na nauunawan ng lahat ng tao sa kahit saanmang sulok ng bansa. Nagkakaroon ng mabisang rehistro ang awitin sa mga tagapakinig dahil hinabi ang liriko nito ayon sa interes ng madla sa kasalukuyang panahon.

4. Mabisa ang pagkakaroon ng tiyak na genre tungo sa mensahe at kahulugan sa mga awitin dahil buong kahusayan itong nakapaghahatid ng layunin sa awitin.

\section{Konklusyon}

Mayaman sa pangwikang pangnilalaman ang mga sinuring awitin na nasa lokal na lenggwahe at nauuso ngayong tugtugin lalong-lalo nasa interes ng mga mag-aaral ito sapagkat ang liriko ng mga awiting ay ang kanilang bernakular na napapadali ang pagkatuto at ugnayang pantao.

\subsection{Rekomendasyon}

Batay sa mga resulta ng pagsusuri, nabuo ang sumusunod na rekomendasyon:

$>\quad$ Bubuo ng gawaing pampagkatuto at pagtuturo ng araling tutugon sa kasanayang pampagkatuto na itinakda ang paglinang ng mga awitin na akma sa kanilang interes at nasa lokal ang mga lilinangin.

$>\quad$ Hikayatin ang mga guro sa Filipino na gamitin ang pinagyamang araling Awiting-bayan gamit ang VARP sa pagsusuri para mapadali ang paghugot sa mga kaisipang ipinapamalas ng mga awitin o sa iba pang akdang pampanitikan.

$>$ Magkaroon ng mga webinar/seminar at worksyap para sa mga guro na magamit ang pagsusuring ginawa sa kanilang pagtuturo sa mga estudayante.

Acknowledgement - All Songs were written by Kuya Bryan at sung by Leela, L.-Pasabta Ko; written and sung by Teodorico, J. -Labyu Langga; written by Ucat, R. and Gitamondoc, J.- Sa Akong Heart; by Ensalada, R. and sung by Von Saw- Pero Atik ra; written by Ucat, R. and Jude Gitamondoc, sung by Fick, K.- HAHAHAHasula. FILSCAP.

This study was presented during the Conference of State Universities and Colleges Teacher Educators Association (SUCTEA) 1st International and Regional Research Conference on June 10-11, 2019 at Bayfront Hotel, Cebu City with the theme: "Multidisciplinary Research in Education: Connect, Collaborate and Innovate". 


\section{Mga sanggunian}

Abangan, V. C., Bercero, R. R., Gera, R. S., \& Magno, O. B. (2009). Filipino 3:Creative expression/rhetoric [Filipino 3: Masining na pagpapahayag/retorika]. Manila: ACCORD Printing Press.

Badayos, P. B. (2008). Methodology in teaching and learning of/in Filipino [Metodolohiya sa pagtuturo at pagkatuto ng/sa Filipino]. Manila: Mutya Publishing House, Incorporated.

Berdin, S. (2007). Poetic analysis of the five selected Cebuano songs that won the first prize in cebu popular music festival [Poetikong pagsusuri sa limangpiling awiting Cebuano na nagwagi ng unang gantimpala sa cebu popular music festival] (MAED unpublished Theses). Cebu Technological University, Cebu City.

Blacking, J. (2019, April 02). How musical is man. https://www.scribd.com/doc/104131606/how-musical-is-man-john-blacking

Brown, K. H. (2019, April 10). Voice, audience, register and purpose (VARP). https://www.slideshare.net/mrLandi/varp.kimhuttonbrown/voice-audienceregister-purpose

Cabangon, N. (2016, September 12). Rico blanco for filipino society of composers, authors and publishers, Incorporated (FILSCAP). Philippine Daily Inquirer. https://entertainment.inquirer.net/218176/rico-blanco-leads-filscap-nurturing-new-generation-composer $\underline{\mathrm{s}}$

Cabangon, N., \& Santos, T. U. (2009). Wika sa saliw ng musika. The Varsitarian Press. https://varsitarian.net/news/20090404/wika_sa_saliw_ng_musika

De Matos, F. (1964). The linguistic sciences and language teaching. Canadian Journal of Linguistics/Revue Canadienne De Linguistique, 10(1), 63-68. https://doi.org/10.1017/S000841310000548X

Fortunato, T. F., \& Valdez, M. S. S. (1995). Pulitika ng wika [Politics of language]. Malate, Manila: De La Salle University Press.

Fuhr, M. (2016). Globalization and popular music in South Korea: Sounding out k-pop. Bulletin of the School of Oriental and African Studies, 79(3), 703-704. https://doi.org/10.1017/S0041977X16000732

Maceda, T. (2007). Problematizing the popular: The dynamics of Pinoy pop(ular) music and popular protest music. Inter-asia Cultural Studies, 8, 390-413. https://doi.org/10.1080/14649370701393766

Macnamara, J. (1967), The bilingual's linguistic performance: A psychological overview. Journal of Social Issues, 23, 58-77. https://doi.org/10.1111/j.1540-4560.1967.tb00576.x

Peregrino, J., Constantino, P. C., \& Ocampo, N. S. (2002). Minanga: Readings on variety and variation of Filipino [Minanga: Mga babasahin sa varayti at varyasyon ng Filipino]. University of the Philippines: Sentro ng Wikang Filipino.

Peregrino, J., Constantino, P. C., \& Ocampo, N. S., \& Petras, J. D. (2012). Salindaw: Variety and variation of Filipino, language in komnet, a new register Filipino language [Salindaw: Varayti at baryasyon ng Filipino, wika sa komnet, isang bagong rehistrong wikang Filipino]. Quezon City: SWF-UPD.

Pesirla, A. (2012). The linguistics of Cebuano binisaya [Ang linggwistika sa sugbuanung binisaya]. Mandaluyong: Anvil Publishing.

Policarpio, A. (March 13, 2017). Rico Blanco leads FILSCAP in nurturing a new generation of composers. Philippine Daily Inquirer.

Rebamonte, G., \& Montera, G. (2007). Filipino poetry [Panulaang Filipino]. Cebu City: Mutya Publishing Incorporated.

Republic Act No. 10533. (2013, September 4). Implementing rules and regulations of the enhanced Basic Education Act of 2013. Official Gazette. https://www.officialgazette.gov.ph/2013/09/04/irr-republic-act-no-10533

Ryback, R. (2019, March 2). Music power explained. https://www.psychologytoday.com/us/blog/thetruismswellness/201601/music-power-explained

Thomason, S. G. (2001). Language and context: A functional linguistic theory of register. London: St. Martin's Press. 
Mandado, J. O., \& Arnado, A. M. M.

36 Consortia Academia Publishing (A partner of Network of Professional Researchers and Educators) 